\title{
Optimizing the power of human performed audio surveys for monitoring the endangered Houston toad using automated recording devices
}

\author{
Andrew R. MacLaren ${ }^{\text {Corresp., } 1,2}$, Paul S. Crump ${ }^{1,3}$, Michael R.J. Forstner ${ }^{1}$ \\ 1 Department of Biology, Texas State University, San Marcos, Texas, United States \\ 2 Cambrian Environmental, Austin, Texas, United States \\ 3 Nongame and rare species program, Texas Parks and Wildlife Department, Austin, Texas, United States \\ Corresponding Author: Andrew R. MacLaren \\ Email address: amaclaren89@gmail.com
}

Knowledge regarding the locations of populations of endangered species is a critical part of recovery and facilitates land use planning that avoids unnecessary impacts. Regulatory agencies often support the development of survey guidelines designed to standardize the methods and maximize the probability of detection, thereby avoiding incorrectly concluding a species is absent from a site. Here, using simulations with data collected using automated recording devices (ARDs) we evaluated the efficacy of the existing U.S. Fish and Wildlife Service's survey requirements for the endangered Houston Toad (Bufo [=Anaxyrus] houstonensis). We explored the effect of 1 ) increasing survey duration, 2) increasing the number of surveys, and 3) combinations of environmental conditions (e.g., temperature, humidity, rainfall) on the detection probability and the number of surveys needed to be $95 \%$ confident of absence. We found that increases in both the duration of the survey and the number of surveys conducted decreased the likelihood of incorrectly concluding the species was absent from the site, and that the number of surveys required to be $95 \%$ confident greatly exceeded the existing survey requirements. Targeting specific environmental conditions was also an effective way to decrease the number of surveys required but the infrequency in which these conditions occurred might make application difficult in some years. Overall, we suggest that the survey effort necessary to achieve confidence in the absence of Houston Toads at a site is more practically achievable with the use of ARDs, but this may not be suitable in all monitoring scenarios. 


\section{Optimizing the Power of Human Performed Audio Surveys}

3 for Monitoring the Endangered Houston Toad Using

4 Automated Recording Devices

Andrew R. MacLaren ${ }^{1,2}$, Paul S. Crump ${ }^{1,3}$, Michael R.J. Forstner ${ }^{1}$

7

8

${ }^{1}$ Department of Biology, Texas State University, San Marcos, Texas, United States

${ }^{2}$ Cambrian Environmental, Austin, Texas, United States

${ }^{3}$ Nongame and Rare Species Program, Texas Parks and Wildlife Department, Austin, Texas, United States

Corresponding Author:

14 Andrew Maclaren ${ }^{1}$

Austin, Texas, United States

Email address: amaclaren89@gmail.com

\section{Abstract}

Knowledge regarding the locations of populations of endangered species is a critical part of recovery and facilitates land use planning that avoids unnecessary impacts. Regulatory agencies often support the development of survey guidelines designed to standardize the methods and maximize the probability of detection, thereby avoiding incorrectly concluding a species is absent from a site. Here, using simulations with data collected using automated recording devices (ARDs) we evaluated the efficacy of the existing U.S. Fish and Wildlife Service's survey requirements for the endangered Houston Toad (Bufo $[=$ Anaxyrus $]$ houstonensis). We explored the effect of 1) increasing survey duration, 2) increasing the number of surveys, and 3) combinations of environmental conditions (e.g., temperature, humidity, rainfall) on the detection probability and the number of surveys needed to be $95 \%$ confident of absence. We found that increases in both the duration of the survey and the number of surveys conducted decreased the likelihood of incorrectly concluding the species was absent from the site, and that the number of surveys required to be $95 \%$ confident greatly exceeded the existing survey requirements. surveys required but the infrequency in which these conditions occurred might make application difficult in some years. Overall, we suggest that the survey effort necessary to achieve confidence in the absence of Houston Toads at a site is more practically achievable with the use of ARDs, but this may not be suitable in all monitoring scenarios.

\section{Introduction}


Monitoring of endangered anuran populations is required to gain an understanding of population dynamics (Pechmann et al. 1991) and the effects of management actions (Walls et al. 2014). Researchers commonly conduct auditory surveys to determine presence or absence of anuran species (Bridges \& Dorcas 2000; Crouch \& Paton 2002; Schmidt 2003; Pierce \& Gutzweiller 2004; Weir et al. 2005; Jackson et al. 2006). Data from these surveys can also be used for estimates of the relative abundance of calling male anurans (Zimmerman 1994) or for determining the cadence or phenology of chorusing behavior (Saenz et al. 2006). These surveys are also used to inform land use and development decisions, in addition to ecological research and endangered species management.

There are presently 14 native anurans, with the inclusion of Puerto Rico, classified by the United States Fish and Wildlife Service (USFWS, hereafter) as threatened and endangered (Anaxyrus californicus, Anaxyrus canorus, Bufo hemiophrys baxterii, Bufo houstonensis, Eleutherodactylus cooki, Eleutherodactylus jasper, Eleutherodactylus juanariveroi, Peltophryne lemur, Rana chiricahua, Rana draytonii, Rana muscosa, Rana pretiosa, Rana sierrae, and Rana sevosa). The USFWS has published guidelines for conducting detection/non-detection surveys for only four of these 14 (B. houstonensis, A. californicus, $R$. chiricahua, and $R$. draytonii; USFWS 1999; USFWS 2005; USFWS 2006; USFWS 2007). Two species ( $R$. pretiosa and $R$. sierrae) have general overviews of currently applied survey methods, authored by the United States Forest Service, but these have not been established as approved policy guidelines. Federal guidelines for conducting surveys of an additional two species (A. canorus and R. muscosa) are reported to be pending approval. The remaining six species lack formal protocols to confirm species presence at a site. These guidelines are intended to ensure that independent researchers are performing standardized surveys designed to maximize the likelihood of detecting the species when present. This lack of survey guidelines, or the existence of poorly designed guidelines, can have serious negative consequences for populations of endangered species. Incorrectly concluding a population of an endangered species is absent from a site can lead to "take" by development of the site through the loss of breeding wetlands and/or upland habitat, as well as potential mortality caused by having individuals present and active in the development site, resulting in preventable losses to the species and expensive fines and protracted delays for the development projects.

Among the existing published guidelines, recommendations for surveying Houston Toads (B. houstonensis) are some of the most specific (USFWS 2007). These guidelines dictate that at minimum, six 5-minute audio surveys, per year, are required at each listening post (i.e. potential breeding location); surveys must be conducted for three consecutive years; surveys should be spread out between February through April; temperatures must be at or above $57^{\circ} \mathrm{F}$; surveys do not begin until about 30 minutes after sundown and cease if a drop in temperature occurs (presumably below $57^{\circ} \mathrm{F}$, however, this is unclear); and wind speeds must not exceed 15 miles per hour (USFWS 2007). These guidelines also include less quantitative recommendations intended to increase the likelihood that Houston Toads are chorusing on nights that are chosen to survey. These include nights in which humidity is greater than $70 \%$; cloud cover is present or the 
79

80

81

82

83

84

85

86

87

88

89

90

91

92

93

94

95

96

97

98

99

100

101

102

103

104

105

106

107

108

109

110

111

112

113

114

115

116

117

118

moon is not full; and rainfall occurring or recent rainfall has occurred. The efficacy of these guidelines has been studied previously; as a result the updated policy now recommends increasing the number of surveys necessary within each season from six to twelve (Jackson et al. 2006; USFWS 2007).

Many studies have revealed the advantages of automated audio recording systems in determining the influential exogenous environmental factors associated with vocalizing behavior when compared to manual call surveys (Bridges \& Dorcas 2000; Oseen \& Wassersug 2002; Hsu et al. 2005; Acevado \& Villanueva-Rivera 2006; Dorcas et al. 2009; Willacy et al. 2015; MacLaren et al. 2018B). In general, automated approaches have the capacity to easily collect significantly more survey data over a wider range of environmental conditions and accordingly provide greater statistical power in examining these relationships over manual surveys. Recently, the use of automated monitoring methods has been more formally recommended for Houston Toad detection/non-detection surveys (USFWS 2020). Thus, the data provided by automated methods provides an excellent opportunity to investigate the true efficacy of manual survey as recommended by the USFWS (2007) guidelines. We are unaware of other studies that model the potential outcomes of a manual survey protocol using data acquired from an automated recording system, but this information is critical to better understand how well the recommended survey guidelines are detecting Houston Toads in particular, and other endangered anurans in general. The use of automated recording methods allows us to investigate the consequences of various important choices in monitoring program design in a systematic and unbiased manner. Thus, the purpose of this study is to 1) evaluate the efficacy of the existing Houston Toad survey guidelines, 2) to investigate any opportunity to improve manual surveys by increasing the duration of each survey and the total number of surveys conducted each year, and 3) to provide updated recommendations for Houston Toad surveys.

\section{Materials \& Methods}

Study Site

We carried out this study utilizing data gathered from the Griffith League Ranch (GLR), located in Bastrop County, Texas, USA. The GLR is a private property owned and operated by the Boy Scouts of America. This property is commonly represented as the primary recovery site for the Houston Toad (Duarte et al. 2014), and received population supplementation through captive propagation efforts both during and prior to when this study was conducted. Audio collected from two Houston Toad breeding locations was used. These sites are separated by 2.37 kilometers and are acoustically independent (see MacLaren et al. 2018C). All work conducted to complete this study was done under scientific permit TE-039544-1 issued by the USFWS.

\section{Audio Recording and Analysis}

We used Song Meter SM3 (Wildlife Acoustics, Maynard, MA) audio recording devices to monitor for the call of male Houston Toads at the two breeding locations on the GLR. Song Meters were programmed to record continuously, beginning in January and ending in July, for 
119 four years (2015-2018). We powered the Song Meters using rechargeable sealed lead acid 120 batteries (Power Sonic PS-6360 NB, 6V, 36.0 AH). We stored the external batteries in plastic

121

122

123

124

125

126

127

128

129

130

131

132

133

134

135

136

137

138

139

140

141

142

143

144

145

146

147

148

149

150

151

152

153

154

155

156

157

158

cases, secured to a structure adjacent to each Song Meter. We equipped each Song Meter with

four $64 \mathrm{~GB}$ SD cards for media storage. The additional costs and data storage requirements associated with continuously monitoring limited us to only two locations. We selected monitoring locations based on their history of maintaining a large number of chorusing male Houston Toads relative to other documented Houston Toad chorusing ponds within the GLR.

To analyze the large quantity of audio files collected, we trained an audio classifier using the software Kaleidoscope version 4.3.1 (Wildlife Acoustics). We followed the steps outlined by the manufacturer for completing this process (Wildlife Acoustics 2017) and used the audio training data provided by MacLaren et al. (2018A) for the call of the Houston Toad. We chose to simply train towards two "clusters", Houston Toad vocalizations, and anything that is not a Houston Toad vocalization. This was efficient, and we achieved 100\% detection of training vocalizations within a single round of training. However, as discussed by MacLaren et al. (2018A) focusing on eliminating false negatives (i.e., a Houston Toad vocalization classified in the "not Houston Toad" group) results in a classifier that is permissible to many false positives that must be manually verified as such. This Kaleidoscope cluster was applied to filter all audio recordings for Houston Toad vocalizations. All detections made by the software were manually verified by ARM. We observed during training that detections below 3 seconds in duration were overwhelmingly false positives, and extremely abundant throughout these data, so we excluded these from Kaleidoscope output prior to manual review. Ultimately, false positive rate for detections greater than 3 seconds in duration was 0.54 , indicating just over half of all detections classified as Houston Toad were not true positives, emphasizing the necessity of manually reviewing all results. We binned detections into 5-minute time intervals, and summarized them as binomial, where 1 and 0 indicate detection and non-detection of Houston Toads, respectively.

\section{$\underline{\text { Simulation }}$}

We simulated Houston Toad audio surveys under three sampling scenarios, each representing a unique approach to subsampling our complete dataset. First, by randomly selecting survey data from the complete pool of recordings from both sites across all years. Second, by restricting available survey data according to environmental conditions presented in the USFWS protocol for conducting surveys for this species (USFWS 2007). Last, we sought to identify the environmental conditions, if any, that maximize the probability of detecting Houston Toads. For environmental variables we used the National Oceanic and Atmospheric Administration's quality controlled local climatic dataset, measured at Giddings, Texas, USA, ca. $25 \mathrm{~km}$ East of our sites (WBAN 53979). We utilized moon illumination measured by the U.S. naval observatory (USNO) for Central Time Zones.

The decision to conduct Houston Toad audio surveys is often made in advance and anticipation of appropriate environmental conditions occurring, based largely on weather forecasts. To reflect the uncertainty implicit in this practice we assumed that if environmental

PeerJ reviewing PDF | (2021:06:62122:1:0:NEW 7 Jul 2021) 
159 thresholds were met at any point within a calendar date, then all data for this date may be

160

161

162

163

164

165

166

167

168

169

170

171

172

173

174

175

176

177

178

179

180

181

182

183

184

185

186

187

188

189

190

191

192

193

194

195

196

197

198 surveyed. This is reflected in the results as "dates surveyable" under each scenario. Each of our three survey scenarios selected only for intervals occurring in the months February, March, and April, before 0600 and after 1800 hours of each date. This not only reflects roughly what is currently required (USFWS 2007), it also coincides with peak chorusing activity for the Houston Toad. To implement a random survey selection scenario, all 5-minute intervals within this time frame were considered. To replicate the restrictions within USFWS (2007) we removed dates wherein environmental variables failed to meet the following thresholds: temperature $>14{ }^{\circ} \mathrm{C}$, relative humidity $>70 \%$, wind speeds $<24 \mathrm{kmph}$, and percent moon illumination $<0.5$. For our final scenario we searched for alternative environmental thresholds by which researchers may improve success when conducting a human-performed audio survey for the Houston Toad. We calculated summary statistics for the following environmental variables, when Houston Toad vocalizations were detected: temperature $\left({ }^{\circ} \mathrm{C}\right)$, relative humidity $(\%)$, wind speed $(\mathrm{kmph})$, moon illumination (\%), hourly precipitation $(\mathrm{mm})$, cumulative precipitation over the previous 24 hours $(\mathrm{mm})$, barometric pressure $(\mathrm{mmHg}$ at sea level), difference in barometric pressure across 24 hours (mmHg at sea level). We then examined which, if any, of these variables offered thresholds that resulted in eliminating the number of dates containing non-detections. We calculated the ratio of detections/non-detections for all combinations of thresholds both above and below all values of temperature, the change in barometric pressure over 24 hours, and cumulative precipitation over the previous 24 hours. This allowed us to identify which thresholds excluded large periods of inactivity within the breeding season. We then applied these thresholds in the same way as described above and carried out the simulation under these new restrictions.

We removed all instances in which the Song Meters did not record, then pooled all Houston Toad detection data (e.g., 5-minute intervals) across the four years and the two sites $(\mathrm{N}=92,652)$. We randomly selected one 5-minute interval for every date $(\mathrm{N}=433)$ without replacement within the pooled dataset and repeated this 1,000 times. This was done to eliminate the possibility of randomly selecting multiple surveys within the same date, which also more correctly reflects how manual surveys are conducted in practice. We calculated the detection probability as the proportion of positive detections out of the total number of files sampled within each permutation, resulting in 1,000 estimates for detection probability for each simulation. From these 1,000 estimates of detection probability, we calculated the mean and $95 \%$ confidence intervals $\left(2.5^{\text {th }}\right.$ and $97.5^{\text {th }}$ quantile) for each distribution of probabilities. We conducted separate simulations for survey scenarios of length 5 minutes, through 60 minutes, by increasing 5-minute intervals, resulting in 12 distributions per scenario. We used the formula provided by Pellet and Schmidt (2005) for calculating the minimum number of surveys required to be $95 \%$ confident in Houston Toad absence, $N_{\min }=\frac{\log (0.05)}{\log (1-p)}$, were $p=$ the simulated estimate of the detection probability for the particular survey scenario.

The detection process consists of many components. First, each site must be occupied during the survey window, then individuals must chorus during the survey window in order for detections to take place. For this study, we assume that for human observers and ARDs this

PeerJ reviewing PDF | (2021:06:62122:1:0:NEW 7 Jul 2021) 
199

200

201

202

203

204

205

206

207

208

209

210

211

212

213

214

215

216

217

218

219

220

221

222

223

224

225

226

227

228

229

230

231

232

233

234

235

236

237

238

detection process is the same. In practice, human observers may disturb chorusing animals leading to higher rates of false-negative errors, but we did not collect comparable data from human observers in order to test for this potential difference in detection.

\section{Results}

We collected detection/non-detection data on 433 dates across all four years $(86,123$, 101, and 123 dates for years 2015-2018, respectively). Out of 92,652 5-minute intervals (intervals, hereafter) we detected Houston Toad vocalizations in 3,975 intervals (approximately 4\%), occurring among 123 dates (18, 35, 37, and 33 dates for years 2015-2018, respectively). Environmental variables measured for the dates which include these intervals are given in Table 1. Only 122 ( $3 \%$ ) of intervals containing Houston Toad vocalizations occurred outside of FebApril. All results henceforth refer to sampling within this peak chorusing period only $(72,359$ total intervals, 328 total dates, 3,853 intervals with detections).

In the first survey scenario, when we selected surveys randomly, the mean detection probability was $0.063(95 \%$ CIs $0.038-0.100)$ for surveys 5 minutes in duration, and 0.121 (95\% CIs $0.088-0.171$ ) for surveys 60 minutes in duration (Figure 1, Table 2). These probabilities result in requiring a mean of 47 (range $=29-79$ ) surveys to be $95 \%$ confident in determining absence of the species when conducting 5-minute-long surveys, and on average 24 (range $=16-33$ ) when conducting 60-minute-long surveys (Figure 1, Table 2).

Our second survey scenario, in which we removed all dates that did not reflect the environmental thresholds given by USFWS (2007), resulted in reducing the number of available survey dates to 118 out of 328 . Under this scenario only 1,737 intervals containing detections are available to be sampled, leaving 1,895 intervals (49\%) known to possess Houston Toad vocalizations unobservable to surveyors $(3,853$ intervals total). The mean detection probability was 0.08 (95\% CIs $0.042-0.119)$ for surveys 5 minutes in duration, and 0.142 (95\% CIs $0.093-$ 0.186 ) for surveys 60 minutes in duration (Figure 1, Table 2). These probabilities result in requiring a mean of 36 (range $=24-70$ ) surveys to be $95 \%$ confident in determining absence of the species when conducting 5-minute-long surveys, and on average 20 (range $=15-31$ ) when conducting 60-minute-long surveys (Figure 1, Table 2).

In our final scenario, calculating the proportion of detections to non-detections over a range of environmental thresholds revealed that a unique combination of temperature $\left(>16^{\circ} \mathrm{C}\right)$, precipitation ( $>0 \mathrm{~mm} /$ day), and change in barometric pressure $(<-0.07 \mathrm{mmHg})$ provided the greatest advantage to surveyors. These thresholds allow 133 dates to be surveyable, comparable to USFWS (2007), yet provide 2,569 intervals containing detections. Under this scenario, the mean detection probability was 0.105 (95\% CIs $0.066-0.146)$ for surveys 5 minutes in duration, and 0.179 (95\% CIs $0.133-0.229$ ) for surveys 60 minutes in duration (Figure 1, Table 2). These probabilities result in requiring a mean of 28 (range $=20-49$ ) surveys to be $95 \%$ confident in determining absence of the species when conducting 5-minute-long surveys, and a mean of 16 (range $=13-22$ ) 60-minute-long surveys to be $95 \%$ confident in determining absence of the species (Figure 1, Table 2).

PeerJ reviewing PDF | (2021:06:62122:1:0:NEW 7 Jul 2021) 


\section{Discussion}

241

242

243

244

245

246

247

248

249

250

251

252

253

254

255

256

257

258

259

260

261

262

263

264

265

266

267

268

269

270

271

272

273

274

275

276

277

278
This study demonstrates that the existing USFWS (2007) guidelines for conducting human performed surveys result in a likelihood of concluding absence of Houston Toads from a site when they may be truly present. Given the endangered status of the species, and the precedent set by Jackson et al. (2006), researchers should attempt to achieve $95 \%$ confidence in conclusions of absence. Currently, 36 (12 per year, for 3 years) 5-minute-long surveys (180 minutes total) are required to determine absence (USFWS 2007). However, our simulation reveals that up to 79 surveys ( 395 total minutes) of this duration may be required to adequately determine Houston Toad occurrence at a single site. Failing to detect Houston Toads when they are truly present ultimately results in undetected populations, which in the event that the monitoring is being performed as part of a development project, may contribute to local extirpation events. This is especially true for populations outside of our study site, which do not receive any form of population supplementation, and may only support a few individuals. It is also important to add that our study, and the study conducted by Jackson et al. (2006), were carried out using data collected from locations where Houston Toads are likely at, or near, their highest abundance, and as such the data presented here could underestimate of the survey requirements necessary to detect other, smaller populations (Tanadini and Schmidt 2011).

Our approach demonstrates that the false negative errors associated with manual surveys can be reduced in three main ways. First, manual surveys of longer duration can be performed. In each of our scenarios, the detection probability increases as survey's duration lengthens. Second, the number of surveys performed can be increased. Third, surveyors can target occasions in which environmental conditions are most closely associated with chorusing behavior among Houston Toads. The trade-offs associated with each of these methods of decreasing false negative errors will depend on the timeframe and scope of the specific Houston Toad monitoring project. Our results indicate human observers must, at minimum, more than double the amount of time spent surveying in order to determine absence with $95 \%$ confidence. However, for human observers these changes may be too onerous to allow manual methods to be feasible for determining detection/non-detection from a site. An alternative approach would be the use of automated recording devices (ARDs), as they are demonstrably effective at the task of detecting anurans, especially when rare, due to the efficiency with which high cumulative detection probabilities are achieved (Hsu et al. 2005; Acevado \& Villanueva-Rivera 2006). Automated audio recording devices designed for monitoring wildlife are becoming smaller, more affordable, and are able to collect audio much more frequently than human observers (Saenz et al. 2006; Aide et al. 2013; Willacy et al. 2015).

The goals of this study, and that of the federal protocol we evaluate (USFWS 2007; USFWS 2020) are limited to determining occurrence of the Houston Toad at a single site (i.e., one human listening post or ARD location). It is our view that human observers should only be employed towards site specific detection of Houston Toads when automated methods are not suitable or potential recorder placement is not permitted. This is particularly relevant for the 
279 Houston Toad as the majority of Texas is privately owned lands and public roadways enable 280 access across remaining habitat patches. Further, human observers might better be employed 281 conducting survey methods that cannot adequately be conducted using remote, passive, methods.

282 For example, in order to detect Houston Toads that do not chorus (i.e., subadults, females)

283 human observers may be required to employ drift fence arrays (Brown et al. 2013) or sample 284 aquatic habitats. Additionally, methods of determining anuran occurrence at larger scales (i.e., 285 county or regional) often require visiting many sites in a single day, and have successfully been 286 implemented using human observers (Gorman 2009). Yantis and Price (1993) employed similar

287

288

289

290

291

292

293

294

295

296

297

298

299

300

301

302

303

304

305

306

307

308

309

310

311

312

313

314

315

316

317 methods to determine the distribution of the Houston Toad within Texas.

One critical aspect in the application of our findings is the frequency with which survey dates containing suitable environmental conditions can be expected to occur. If we consider the four years utilized in this study (2015-2018), we find that conditions permissible under the currently accepted USFWS (2007) guidelines only occur on 27, 39, 39, and 31 dates within peak chorusing period each year, respectively. Our simulation indicates that up to 79 5-minute-long surveys should be conducted to minimize false negative errors, and accordingly surveyors would be required to choose an alternative approach (i.e., surveys of longer duration) in order to achieve confidence in their findings. If we consider the environmental conditions discussed in our third scenario, we find that for years 2015-2018, these conditions only occur on 40,53,5, and 50 dates within the peak chorusing period each year, respectively. While these results illustrate that in certain years this increases the abundance of surveyable days, during 2017 this method provides only five days of survey appropriate conditions, which reduces the practicality of this approach considerably. Additionally, it would be unwise for researchers to restrict their survey design to a limited set of environmental conditions when these events occur stochastically, and are not guaranteed to occur the necessary number of times to have confidence in conclusions of absence. A caveat within this scenario is that the range of conditions we identified during are derived from our complete dataset, and have not been applied to an independent group of data, and so our results from this scenario may be biased optimistically.

Given the environmental and temporal constraints we have identified, researchers have much to consider when scheduling acoustic surveys for the Houston Toad. The cost to researchers performing acoustic surveys using human observers is likely variable, and we will not speculate on the individual cost of each survey. Although ARDs are, in the long term, less expensive than human performed surveys, factors such as travel and wages can be minimized (Williams et al. 2018). We believe our approach demonstrating detection probability as it increases with survey duration provides researchers under variable circumstances options to achieve confidence in absence determinations. For example, if travel costs are large (i.e., researchers must travel a great distance to reach their field sites) perhaps fewer surveys of greater duration may be favored, whereas researchers with many sites to visit may prefer shorter surveys, allowing more sites to be visited on each calendar date.

\section{Conclusions}


Given the findings of our simulations, we strongly recommend that human observers restrict their surveys to the peak of Houston toad activity that occurs during the 89 day period between February 1 and April 30. While we believe it is wise to use a priori knowledge of the environmental conditions in which chorusing generally takes place to improve the likelihood of detecting Houston Toads (MacLaren et al. 2018B), our study reveals that these events are rare, do not consistently elicit vocalization behavior, and may not allow for adequate effort to be put forth by human observers in any given year. For these reasons we feel our first scenario is most applicable, in which surveyors can choose to survey any date. Houston Toads are in decline throughout their native range (Forstner and Dixon 2011) thus, we believe that, due to the serious consequences of potential false negative errors, the upper $95 \%$ confidence interval for randomly selected surveys be adopted as the minimum survey effort threshold (Table 2). Situations that trigger the need to conduct surveys following USFWS $(2007 ; 2020)$ are likely to occur in areas where local occurrence is not known (e.g., marginal habitats). Marginal populations are in the most need of stewardship, and maximum survey effort (i.e., the upper bounds of our confidence intervals, or beyond) is likely necessary to detect these less abundant population remnants.

We found that previously suggested environmental correlates to chorusing among Houston Toads offered improved detection probabilities over randomly selected surveys. However, we found that not all suggested weather criteria within USFWS (2007) were useful, specifically, moon illumination, humidity, and wind speed. This is either because these variables share no true relationship with chorusing within Houston Toads, as is the case for moon illumination, or because they do not serve as a hard boundary, as is the case for relative humidity. For example, relative humidity may range between $10 \%$ and $90 \%$ within a given single date in response to natural diel cycle. We identified definitive thresholds among temperature, precipitation, and shifts in barometric pressure that improve the probability of detection for Houston Toads beyond what USFWS (2007) currently suggests.

This study updates and expands upon the findings of Jackson et al. (2006). For perspective this previous study (Jackson et al. 2006) utilized twenty 5-minute surveys (100 minutes) within a given year at a single site, whereas within a single year one ARD provided us with approximately 60,000 minutes of audio from a single site. Using these vast and detailed data we found that detection probabilities, for surveys of any length, and under any sampling scenario, were lower than what has been previously estimated for this species (Jackson et al. 2006). By suggesting more accurate environmental thresholds under which surveys should be conducted, and evaluating surveys of varying duration, we have provided researchers and managers with an approach that should make the highest probability of detecting Houston Toads possible. Our approach to simulating survey effort allows researchers to choose the combination of survey duration and number of surveys they find most appropriate and maintain $95 \%$ confidence in determinations of absence. Like Jackson et al. (2006) our results suggest that the USFWS should modify the mandatory survey guidelines to require more surveys in each season than is currently specified. Moreover, for surveys that are designed to determine presence or absence towards regulatory decisions at a site, the conflict between the availability of suitable 
359

360

361

362

363

364

365

366

367

368

369

370

371

372

373

374

375

376

377

378

379

380

381

382

383

384

385

386

387

388

389

390

391

392

393

394

395

396

397

398

environmental conditions and the importance of conducting sufficient surveys based on environmental factors that increase the probability of survey success, ARDs should be strongly considered where possible. Finally, it is critical to differentiate absence determinations made from chorusing data from true absence of this endangered anuran from a potential disturbance site given the underlying nature of juvenile amphibian dispersal and adult use of upland habitats.

\section{Acknowledgements}

We thank the Capitol Area Council-Boy Scouts of America for their cooperation, and Shawn McCracken, Floyd Weckerly, Joe Veech, Andy Royle, Ben Bolker, and Charles Hermann for reviewing earlier drafts of this work.

\section{References}

Acevedo, M.A. and Villanueva-Rivera, L.J., 2006. Using Automated Digital Recording Systems as Effective Tools for the Monitoring of Birds and Amphibians. Wildlife Society Bulletin, 34(1), pp.211-214.

Aide, T.M., Corrada-Bravo, C., Campos-Cerqueira, M., Milan, C., Vega, G. and Alvarez, R., 2013. Real-time bioacoustics monitoring and automated species identification. PeerJ, 1 , p.e103.

Alldredge, M.W., Pollock, K.H., Simons, T.R., Collazo, J.A. and Shriner, S.A., 2007. Time-ofdetection method for estimating abundance from point-count surveys. The Auk, 124(2), pp.653-664.

Bridges, A.S. and Dorcas, M.E., 2000. Temporal variation in anuran calling behavior: implications for surveys and monitoring programs. Copeia, 2000(2), pp.587-592.

Brown, D.J., Duarte, A., Mali, I., Jones, M.C. and Forstner, M.R., 2014. Potential impacts of a high severity wildfire on abundance, movement, and diversity of herpetofauna in the Lost Pines ecoregion of Texas. Herpetological Conservation and Biology, 9(1), pp.192-205.

Brown, D.J., Swannack, T.M. and Forstner, M.R., 2013. Predictive models for calling and movement activity of the endangered Houston toad. The American Midland Naturalist, 169(2), pp.303-321.

Charif, R. and Pitzrick, M., 2008. Automated detection of Cerulean Warbler songs using XBAT data template detector software. Preliminary Report. Bioacoustics Research Program Cornell Laboratory of Ornithology Technical Report, pp.08-02.

Crouch, W.B. and Paton, P.W., 2002. Assessing the use of call surveys to monitor breeding anurans in Rhode Island. Journal of Herpetology, 36(2), pp.185-193.

Dorcas, M.E., Price, S.J., Walls, S.C. and Barichivich, W.J., 2009. Auditory monitoring of anuran populations. Amphibian ecology and conservation: a hand book of techniques. Oxford University Press, Oxford, pp.281-298.

Duarte, A., Brown, D.J. and Forstner, M.R., 2014. Documenting extinction in real time: decline of the Houston toad on a primary recovery site. Journal of Fish and Wildlife Management, 5(2), pp.363-371. 
399

400

401

402

403

404

405

406

407

408

409

410

411

412

413

414

415

416

417

418

419

420

421

422

423

424

425

426

427

428

429

430

431

432

433

434

435

436

437

Forstner, M.R.J., and J.R. Dixon. 2010. Five-year status review: Houston toad. Final report for Section 6 project \#E-101-R. 64pgs.

Gaston, M.A., Fuji, A., Weckerly, F.W. and Forstner, M.R., 2010. Potential component allee effects and their impact on wetland management in the conservation of endangered anurans. PLoS One, 5(4), p.e10102.

Gorman, T.A., 2009. Ecology of two rare amphibians of the Gulf Coastal Plain (Doctoral dissertation, Virginia Tech).

Hsu, M.Y., Kam, Y.C. and Fellers, G.M., 2005. Effectiveness of amphibian monitoring techniques in a Taiwanese subtropical forest. The Herpetological Journal, 15(2), pp.7379.

Hutto, R.L. and Stutzman, R.J., 2009. Humans versus autonomous recording units: A comparison of point-count results. Journal of Field Ornithology, 80(4), pp.387-398.

Jackson, J.T., Weckerly, F.W., Swannack, T.M. and Forstner, M.R.J., 2006. Imperfect detection and number of auditory surveys for Houston toads. J. Wildlife Management, 70, pp.14611463.

MacLaren, A.R., McCracken, S.F. and Forstner, M.R., 2018A. Development and Validation of Automated Detection Tools for Vocalizations of Rare and Endangered Anurans. Journal of Fish and Wildlife Management, 9(1), pp.144-154.

MacLaren, A.R., McCracken, S.F. and Forstner, M.R., 2018B. Automated Monitoring Techniques Reveal New Proximate Cues of Houston Toad Chorusing Behavior. Herpetological Review, 49(4), pp.622-626.

MacLaren, A.R., Crump, P.S., Royle, J.A. and Forstner, M.R., 2018C. Observer-free experimental evaluation of habitat and distance effects on the detection of anuran and bird vocalizations. Ecology and evolution, 8(24), pp.12991-13003.

Oseen, K.L. and Wassersug, R.J., 2002. Environmental factors influencing calling in sympatric anurans. Oecologia, 133(4), pp.616-625.

Pechmann, J.H., Scott, D.E., Semlitsch, R.D., Caldwell, J.P., Vitt, L.J. and Gibbons, J.W., 1991. Declining amphibian populations: the problem of separating human impacts from natural fluctuations. Science, 253(5022), pp.892-895.

Pellet, J. and Schmidt, B.R., 2005. Monitoring distributions using call surveys: estimating site occupancy, detection probabilities and inferring absence. Biological Conservation, 123(1), pp.27-35.

Pierce, B. and Gutzweiller, K., 2004. Auditory sampling of frogs: detection efficiency in relation to survey duration. Journal of Herpetology, 38(4), pp.495-500.

R Development Core Team, 2018. R: A language and environment for statistical computing. Vienna, Austria: R Foundation for Statistical Computing.

Rempel, R.S., Francis, C.M., Robinson, J.N. and Campbell, M., 2013. Comparison of audio recording system performance for detecting and monitoring songbirds. Journal of Field Ornithology, 84(1), pp.86-97. 
438

439

440

441

442

443

444

445

446

447

448

449

450

451

452

453

454

455

456

457

458

459

460

461

462

463

464

465

466

467

468

469

470

471

472

473

474

475

476

477

Saenz, D., Fitzgerald, L.A., Baum, K.A. and Conner, R.N., 2006. Abiotic correlates of anuran calling phenology: the importance of rain, temperature, and season. Herpetological Monographs, 20(1), pp.64-82.

Schmidt, B.R., 2003. Count data, detection probabilities, and the demography, dynamics, distribution, and decline of amphibians. Comptes Rendus Biologies, 326(1), pp.119-124.

Tanadini, L.G. and B.R. Schmidt. 2011. Population size influences amphibian detection probability: Implications for biodiversity monitoring programs. PLoS ONE 6(12), p.e28244.

[USFWS] U.S. Fish and Wildlife Service, U.S.F.W.S. 1999. Survey Protocol for the Arroyo Toad. U.S. Fish and Wildlife Service, Carlsbad \& Ventura, California.

[USFWS] U.S. Fish and Wildlife Service, U.S.F.W.S. 2005. Revised Guidance on Site Assessments and Field Surveys for the California Red-legged Frog. U.S. Fish and Wildlife Service, Sacramento, California.

[USFWS] U.S. Fish and Wildlife Service, U.S.F.W.S. 2006. Chiricahua Leopard Frog (Rana chiricahua) Draft Recovery Plan with appendices. Appendix E. E-1 - E-15 pp.

[USFWS] U.S. Fish and Wildlife Service, U.S.F.W.S. 2007. Section 10(a)(1)(A) Scientific permit requirements for conducting Houston Toad presence/absence surveys. U.S. Fish and Wildlife Service, Austin, Texas.

[USFWS] U.S. Fish and Wildlife Service, U.S.F.W.S. 2020. Section 10(a)(1)(A) Scientific permit requirements for conducting Houston Toad presence/absence surveys, Version 1.1. U.S. Fish and Wildlife Service, Austin, Texas.

Walls, S.C., J.H. Waddle, W.J. Barichivich, I.A. Bartoszek, M.E. Brown, J.M. Hefner, and M.J. Schuman. 2014. Anuran site occupancy and species richness as tools for evaluating restoration of a hydrologically-modified landscape. Wetlands Ecology and Management 22(6), pp.625-639.

Weir, L., and Mossman, M.J., 2005. North American Amphibian Monitoring Program (NAAMP). In Amphibian Declines: The Conservation Status of United States Species. University of California Press, Berkeley, California, USA. pp.307-313.

Weir, L., Fiske, I.J. and Royle, J.A., 2009. Trends in anuran occupancy from northeastern states of the North American Amphibian Monitoring Program. Herpetological Conservation and Biology, 4(3), pp.389-402.

Wildlife Acoustics, 2017. Kaleidoscope 4.3.1 user manual. Maynard, Massachusetts: Wildlife Acoustics.

Williams, E.M., O'Donnell, C.F. and Armstrong, D.P., 2018. Cost-benefit analysis of acoustic recorders as a solution to sampling challenges experienced monitoring cryptic species. Ecology and evolution, 8(13), pp.6839-6848.

Yantis, J.H., and Price, A.H., 1993. Final report. As required by the Endangered Species Act, Section 6. Texas, Project No: E-1-4. Endangered and Threatened Species Conservation, Job No. 8, Houston Toad (Bufo houstonensis) status survey. Texas Parks and Wildlife Department, Austin TX.

PeerJ reviewing PDF | (2021:06:62122:1:0:NEW 7 Jul 2021) 
478 Zimmerman, B.L., 1994. Audio strip transects. In measuring and monitoring biological diversity:

479 standard methods for amphibians. Smithsonian Institution Press, Washington, D.C., $480 \quad$ USA. pp. 92-97. 


\section{Table $\mathbf{1}$ (on next page)}

Summary statistics of environmental variables during dates in which Houston Toads (Bufo houstonensis) were detected by automated audio recorders from 2015-2018 in Bastrop County, Texas. 
1 Table 1. Summary statistics of environmental variables during dates in which Houston Toads

2 (Bufo houstonensis) were detected by automated audio recorders from 2015-2018 in Bastrop

3 County, Texas.

4

\begin{tabular}{l|rrrrrr} 
& MIN. & 1ST QU. & MEDIAN & MEAN & 3RD QU. & MAX. \\
\hline TEMPERATURE $\left({ }^{\circ} \mathrm{C}\right)$ & -1.15 & 16.63 & 19.20 & 19.24 & 22.00 & 31.40 \\
RELATIVE HUMIDITY (\%) & 14.33 & 72.33 & 88.00 & 82.63 & 95.67 & 100.00 \\
WIND SPEED (KMPH) & 0.00 & 3.67 & 7.00 & 7.19 & 9.67 & 28.33 \\
BAROMETRIC PRESSURE (MMHG) & 29.06 & 29.38 & 29.47 & 29.47 & 29.57 & 29.99 \\
PRESSURE CHANGE (MMHG) & -0.35 & -0.11 & -0.04 & -0.03 & 0.04 & 0.38 \\
PRECIPITATION (MM) & 0.00 & 0.03 & 0.07 & 0.24 & 0.22 & 4.50 \\
MOON ILLUMINATION (\%) & 0.00 & 11.00 & 40.00 & 48.76 & 91.00 & 100.00
\end{tabular}

5 


\section{Table 2 (on next page)}

Raw values of probability of detecting Houston Toads, and the number of surveys needed to infer absence, as duration of survey increases.

Mean and $95 \%$ confidence bounds for the probability of detection and number of surveys required to be $95 \%$ confident in absence of Houston Toads (Bufo houstonensis) during acoustic surveys, for three sampling paradigms, as duration of survey increases from 5 to 60 minutes. 
1 Table 2. Mean and 95\% confidence bounds for the probability of detection and number of 2 surveys required to be $95 \%$ confident in absence of Houston Toads (Bufo houstonensis) during 3 acoustic surveys, for three sampling paradigms, as duration of survey increases from 5 to 60 4 minutes.

\begin{tabular}{cccc|ccc} 
& \multicolumn{3}{c}{ Detection Probability } & \multicolumn{3}{c}{ Number of Surveys } \\
\cline { 2 - 7 } $\begin{array}{c}\text { Duration } \\
\text { (mins) }\end{array}$ & $2.50 \%$ & Mean & $97.50 \%$ & $2.50 \%$ & Mean & $97.50 \%$ \\
\cline { 2 - 7 } & & & Randomly Selected & & \\
\hline 5 & 0.038 & 0.063 & 0.100 & 79 & 47 & 29 \\
10 & 0.046 & 0.074 & 0.113 & 64 & 39 & 26 \\
15 & 0.050 & 0.082 & 0.121 & 59 & 35 & 24 \\
20 & 0.058 & 0.089 & 0.129 & 50 & 33 & 22 \\
25 & 0.063 & 0.094 & 0.138 & 47 & 31 & 21 \\
30 & 0.067 & 0.099 & 0.142 & 44 & 29 & 20 \\
35 & 0.071 & 0.104 & 0.146 & 41 & 28 & 19 \\
40 & 0.075 & 0.107 & 0.150 & 39 & 27 & 19 \\
45 & 0.075 & 0.111 & 0.154 & 39 & 26 & 18 \\
50 & 0.079 & 0.115 & 0.158 & 37 & 25 & 18 \\
55 & 0.083 & 0.118 & 0.167 & 35 & 24 & 17 \\
60 & 0.088 & 0.121 & 0.171 & 33 & 24 & 16 \\
\hline & & & USFWS Protocol & & \\
\hline \hline 5 & 0.042 & 0.080 & 0.119 & 70 & 37 & 24 \\
10 & 0.051 & 0.094 & 0.136 & 58 & 31 & 21 \\
15 & 0.059 & 0.103 & 0.153 & 49 & 28 & 19 \\
20 & 0.068 & 0.110 & 0.153 & 43 & 26 & 19 \\
25 & 0.076 & 0.116 & 0.161 & 38 & 25 & 18 \\
30 & 0.076 & 0.121 & 0.169 & 38 & 24 & 17 \\
35 & 0.085 & 0.126 & 0.169 & 34 & 23 & 17 \\
40 & 0.085 & 0.129 & 0.178 & 34 & 22 & 16 \\
45 & 0.085 & 0.133 & 0.178 & 34 & 21 & 16 \\
50 & 0.093 & 0.136 & 0.178 & 31 & 21 & 16 \\
55 & 0.093 & 0.140 & 0.186 & 31 & 20 & 15 \\
60 & 0.093 & 0.143 & 0.186 & 31 & 20 & 15 \\
\hline & & & $0 p 16$ & & \\
\hline \hline 5 & 0.060 & 0.101 & 0.143 & 49 & 28 & 20 \\
10 & 0.075 & 0.118 & 0.165 & 39 & 24 & 17 \\
15 & 0.083 & 0.128 & 0.173 & 35 & 22 & 16 \\
20 & 0.090 & 0.136 & 0.180 & 32 & 21 & 16 \\
25 & 0.098 & 0.143 & 0.188 & 30 & 20 & 15 \\
30 & 0.105 & 0.149 & 0.195 & 27 & 19 & 14 \\
35 & 0.113 & 0.154 & 0.195 & 25 & 18 & 14 \\
40 & 0.113 & 0.159 & 0.203 & 25 & 18 & 14 \\
45 & 0.120 & 0.163 & 0.203 & 24 & 17 & 14 \\
50 & 0.120 & 0.167 & 0.211 & 24 & 17 & 13 \\
55 & 0.128 & 0.170 & 0.211 & 22 & 17 & 13 \\
60 & 0.128 & 0.173 & 0.218 & 22 & 16 & 13 \\
\hline \hline & & & & & & \\
\hline
\end{tabular}




\section{Figure 1}

Probability of detecting Houston Toads, and the number of surveys needed to infer absence, as duration of survey increases.

Results of simulation to assess mean probability of detection of Houston Toads (Bufo houstonensis) (bottom panel), and the mean number of surveys necessary for a given probability of detection (top panel), and their $95 \%$ confidence bounds, as the length in minutes of each auditory survey increases along the x-axis, for three approaches to survey selection: Random selection (left), following USFWS 2007 (middle), and under our proposed optimization for survey selection (right).

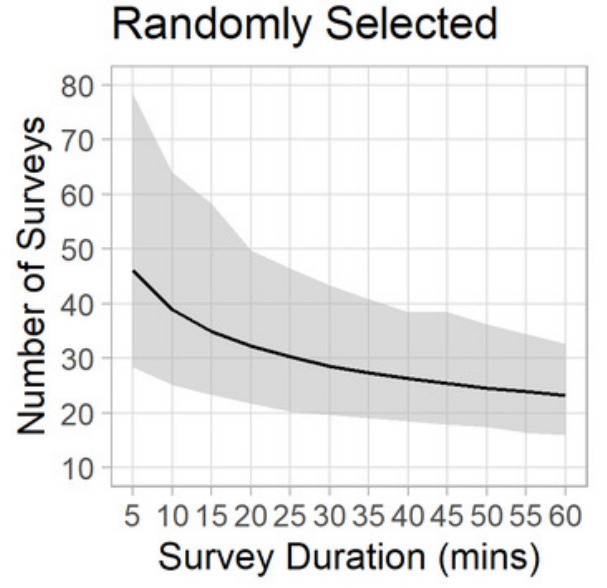

Randomly Selected

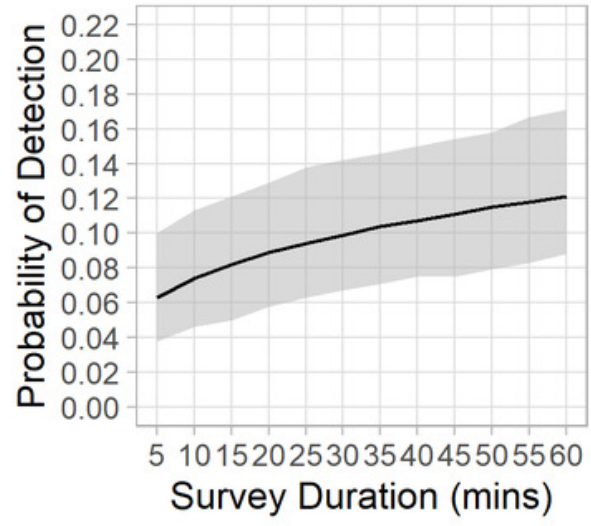

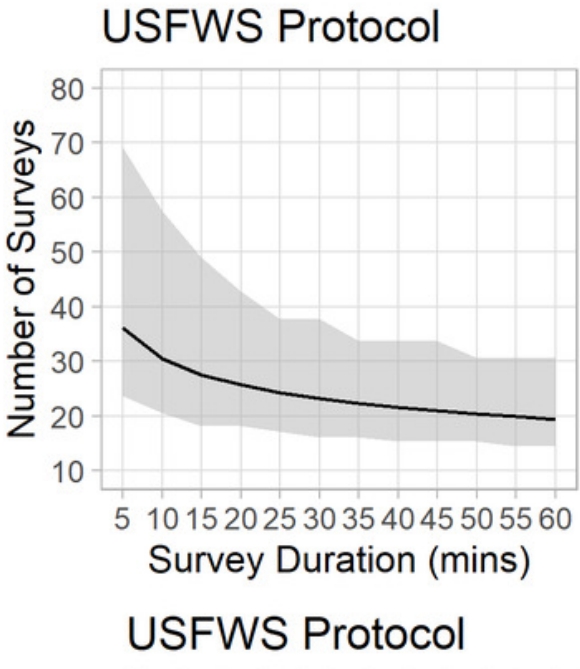

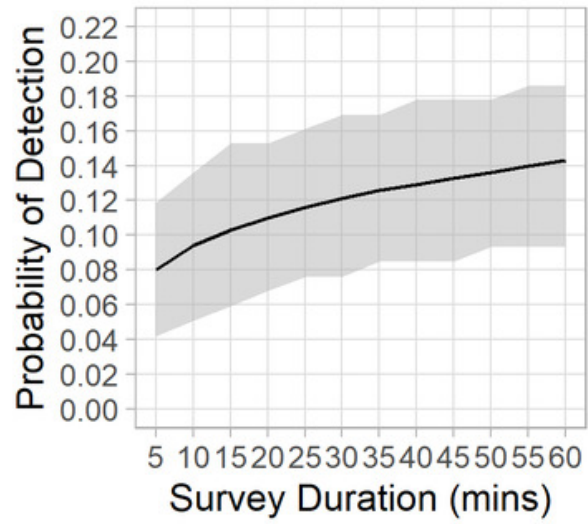

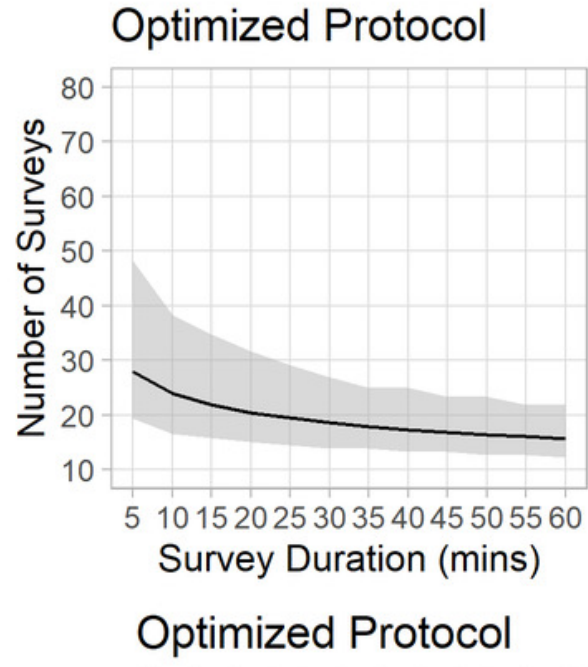

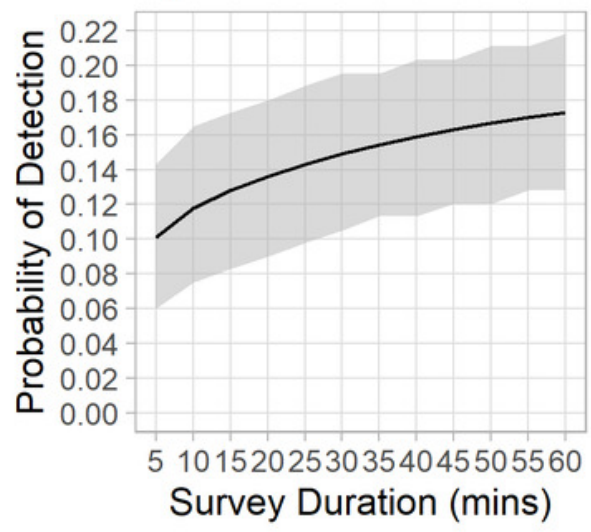

